\title{
Genetic Variability, Evolution, and Biological Effects of Grapevine fanleaf virus Satellite RNAs
}

\author{
J. Gottula, D. Lapato, K. Cantilina, S. Saito, B. Bartlett, and M. Fuchs
}

Department of Plant Pathology and Plant-Microbe Biology, Cornell University, New York State Agricultural Experiment Station, Geneva, NY 14456.

Current address for D. Lapato: Quest Diagnostics Laboratory, 14225 Newbrook Drive, Chantilly, VA 20151.

Current address for S. Saito: USDA-ARS San Joaquin Agricultural Sciences Center, 9611 South Riverbend Avenue, Parlier, CA 93648.

Current address for B. Bartlett: Mississippi Genome Exploration Laboratory and Department of Plant and Soil Sciences, Mississippi State University, MS 39762.

Accepted for publication 23 May 2013.

\begin{abstract}
Gottula, J., Lapato, D., Cantilina, K., Saito, S., Bartlett, B., and Fuchs, M. 2013. Genetic variability, evolution, and biological effects of Grapevine fanleaf virus satellite RNAs. Phytopathology 103:1180-1187.

Large satellite RNAs (type B satRNAs) of Grapevine fanleaf virus (GFLV) from the genus Nepovirus, family Secoviridae were identified in a naturally infected vineyard and a grapevine germplasm collection. These GFLV satRNA variants had a higher nucleotide sequence identity with satRNAs of Arabis mosaic virus (ArMV) strains NW and J86 (93.8 to $94.6 \%$ ) than with the satRNA of GFLV strain F13 and those of other

(GFLV, ArMV, and Grapevine deformation virus) and nepovirus type B satRNAs, including previously reported motif I, suggesting that large satRNAs might have originated from recombination between an ancestral subgroup A nepovirus RNA and an unknown RNA sequence with the $5^{\prime}$ region acting as a putative cis-replication element. A comparative analysis of two GFLV strains carrying or absent of satRNAs showed no discernable effect on virus accumulation and symptom expression in Chenopodium quinoa, a systemic herbaceous host. This work sheds light on the origin and biological effects of large satRNAs associated with subgroup A nepoviruses.
\end{abstract} ArMV strains (68.3 to $75.0 \%$ ). Phylogenetic analyses showed no distinction of GFLV and ArMV satRNAs with respect to the identity of the helper virus. Seven stretches of 8 to 15 conserved nucleotides (I-VII) were identified in the $5^{\prime}$ region of subgroup A nepovirus genomic RNAs
Additional keywords: commensalism, genetic distance, plant virus symptoms, Vitis vinifera.
Grapevine fanleaf virus (GFLV) is the primary causal agent of fanleaf degeneration disease of grapevine. This virus causes severe economic losses worldwide (2). Arabis mosaic virus (ArMV) and Grapevine deformation virus (GDefV) also cause fanleaf degeneration in central Europe (28). GFLV, ArMV and GDefV are subgroup A members of the genus Nepovirus in the family Secoviridae and have similar bipartite single-stranded RNA genomes (36). GFLV and ArMV are closely related $(2,36)$ and GDefV may result from recombination between GFLV and ArMV $(6,10)$.

Nepoviruses, including some GFLV and ArMV isolates, contain large and small satellite RNAs (satRNAs) (7). Large satRNAs, which tend to be greater than $1 \mathrm{~kb}$ and have an open reading frame (ORF), are referred to as type B satRNAs (29). They are absolutely dependent on a helper genome for replication and encapsidation, and encode a nonstructural protein. Little is known about the origin and function of nepovirus type $B$ satRNAs or their encoded protein. The satRNA associated with GFLV strain F13 is 1,114 nucleotides (nts) long and encodes a 37-kDa protein called P3. This is the only large satRNA characterized so far for GFLV $(9,13,30,33)$ although a new GFLV satRNA was recently reported (20). SatRNAs of ArMV range from 1,092 to $1,139 \mathrm{nts}$ in size and produce a protein of $39 \mathrm{kDa}$

Corresponding author: M. Fuchs; E-mail address: mf13@ cornell.edu

* The $\boldsymbol{e}$-Xtra logo stands for "electronic extra" and indicates that the online version contains one supplemental table.

http://dx.doi.org/10.1094/PHYTO-11-12-0310-R

(c) 2013 The American Phytopathological Society
$(27,43)$. SatRNAs associated with three ArMV isolates from Neustadt an der Weinstrasse (NW) in Germany are 99\% similar at the nucleotide level, while satRNAs associated with other isolates differ greatly, showing as low as 57\% amino acid and $73 \%$ nucleotide identity to NW (43). Interestingly, ArMV-NW satRNAs have slightly higher identity to the GFLV-F13 satRNA than to some other ArMV satRNAs (43). Replication of nepovirus satRNAs by helper viruses is achieved with some degree of specificity. The ArMV satRNA can be replicated by satRNAdeficient ArMV strains Ash and Ivy but not by ArMV strains Hop or AB10 or the genome of other nepoviruses, including GFLV (24). The Tomato black ring virus (TBRV) satRNAs replicate only with certain isolates of TBRV, owing specificity to either a helper virus-encoded factor (7) or the protein encoded by the satRNA $(14,32)$. In contrast, the satRNA of GFLV-F13 replicates in Chenopodium quinoa plants infected with satRNA-deficient GFLV strain TU $(33)$ or ArMV $(8,13)$. SatRNAs are not known to have similarity to sequences available in GenBank, except a short sequence conserved within the $5^{\prime}$ end of nepoviruses (9).

There is no clear association of nepovirus type B satRNAs and viral virulence $(3,34)$. For example, symptoms of GFLV-infected grapevines do not seem to be influenced by presence or absence of satRNAs (35). On model hosts, information on satRNAinduced symptoms is contradictory, with some studies failing to detect an association (7) and others showing an effect on symptoms and virus accumulation in a host species-dependent manner. For example, the ArMV-lilac satRNA does not promote significant differences in ArMV titer in C. quinoa, but prevents virus-induced tip necrosis (25). An experiment using GFLV strains devoid of satRNAs, to which transcripts of the satRNA of 
GFLV-F13 were added, suggested a slight delay (1 to 2 days) in symptom development in $C$. quinoa (8), but this study did not rely on GFLV strains with a homogenous genetic background.

The type B satRNA is fairly prevalent in GFLV or ArMV isolates. Surveys of grapevine collections or virus cultures acquired from geographically diverse origins detected a satRNA in 5 out of 34 GFLV-infected samples by RNA hybridization (35), and in 6 of 38 ArMV-infected samples using reverse transcriptionpolymerase chain reaction (RT-PCR) (43). No information is available on the occurrence and distribution of type B satRNA in naturally infected commercial vineyards. This study addresses the GFLV satRNA origin, epidemiology, genetic variability, and effect on helper virus multiplication and symptomatology. Our aim was to characterize satRNAs in a naturally GFLV-infected vineyard, compare their genetic structure to those of known GFLV and ArMV satRNAs and genomic RNAs, and determine their effect on GFLV virulence on the model host $C$. quinoa.

\section{MATERIALS AND METHODS}

Plant material. Grapevine leaf samples were collected on 14 May 2010 and another set on 10 May 2012 in Lodi, CA. Fifty nine samples were taken from a vineyard that contained a mixture of rootstock genotypes. These vines were established on a site where Vitis vinifera 'Zinfandel' scions grafted onto Freedom (1613-59 $\times$ Dog Ridge) rootstocks were previously grown (31). Samples were also taken in 2010 from two nearby vineyards, including 16 samples from a vineyard of $V$. vinifera 'Zinfandel' scions grafted onto Freedom rootstocks, and eight samples from a vineyard of $V$. vinifera 'Cabernet Sauvignon' scions grafted onto Dog Ridge (V. champini) rootstocks. Each vineyard was naturally infested with GFLV-viruliferous Xiphinema index and ArMV was not present (31). Leaf samples were also collected on 15 June 2010 on GFLV-infected vines at the cold-hardy grape germplasm collection, USDA-Plant Genetic Resource Unit (PGRU), Geneva, NY.

GFLV and satRNA detection by ELISA and IC-RT-PCR. Double-antibody sandwich (DAS) enzyme-linked immunosorbent assay (ELISA) and immunocapture-reverse transcription (IC-RT) polymerase chain reaction (PCR) was conducted with specific GFLV antibodies (Bioreba Inc., Reinach, Switzerland). For ELISA, absorbance at $\mathrm{OD}_{405 \mathrm{~nm}}$ was read by a Synergy 2 microplate reader and analyzed using Gen5 software (Biotek Corporation, Winooski, VT). The mean absorbance of two in-plate replications for each ELISA sample value was taken. Absorbance values of test samples were blanked by subtracting the absorbance value of a GFLV-free grape leaf or $C$. quinoa leaf extract.

Reverse transcription was conducted following capture of GFLV virions (40) using AMV reverse transcriptase and an 18mer poly-T primer (New England Biolabs, Ipswich, MA) following manufacturer's protocol. PCR was conducted on cDNA with GoTaq PCR mixture (Promega, Southampton, UK). The GFLV satRNA was detected using primers NepSatF and NepSatR, and GFLV RNA2 was detected using primers G34 and G37 (Supplemental Table 1). All DNA amplicons were size fractioned by electrophoresis on a $1.5 \%$ agarose gel in a TAE buffer. Positive controls included the satRNA of GFLV strain F13 (33) and isolate R3 from Lodi, CA (this study). Negative controls for PCR included a water control in place of RNA template, and GFLV-free grape or $C$. quinoa cDNA produced from the IC-RT step.

$5^{\prime}$ Rapid amplification of cDNA ends (RACE) and $3^{\prime}$ amplification. A 5' RACE procedure and $3^{\prime}$ amplification were carried out to determine the nucleotide sequence of the termini of GFLV satRNA sequences. Immunocapture was obtained from extracts of C. quinoa plants infected with satRNA-containing GFLV strains R6-40 and R2-39, as well as satRNA negative GFLV strains R618 (this study) and FF, the later being obtained from in vitro transcripts of GFLV-F13 RNA1 and RNA2 cDNAs (41). Complementary DNA was synthesized as described above with poly-T primers (for 3' amplification) or NepSatR (for 5' RACE). For 5' RACE, dATP was joined to the $3^{\prime}$ ends of cDNAs with terminal deoxynucleotide transferase (New England Biolabs) and products were amplified by PCR first with oligodT-target and SP1, and then with TargetF (corresponding to the specific sequence in oligo-dT-target) and SP2. Initial 3' amplification was conducted with oligo-dT-target and NepSatF, followed by a second round of PCR with NepSatF and TargetF.

Cloning and sequencing PCR amplicons of GFLV genomic RNA and satRNAs. Size-fractioned PCR products were extracted from gels using an Omega Gel Extraction Kit (Omega Biotek, Doraville, GA), T/A cloned into PCR4-TOPO (Invitrogen, Carlsbad, CA) and transformed into chemically competent TOP10 E. coli. Plasmids were extracted from single colony transformants with Plasmid Mini-Prep Kit (Omega) and digested with EcoRI (New England Biolabs) to confirm expected size fractions. Sequences were generated via Sanger sequencing with the M13 primer sets at the Cornell University Life Sciences Core Laboratories Center and full bidirectional coverage was obtained for each TOPO-cloned insert.

Phylogenetic trees and genetic analyses. Alignments for GFLV and ArMV genes $1 \mathrm{E}^{\mathrm{Pol}}, 2 \mathrm{~A}^{\mathrm{HP}}, 2 \mathrm{~B}^{\mathrm{MP}}, 2 \mathrm{C}^{\mathrm{CP}}$, and satRNAs were created using all full-length or almost full-length sequences available in GenBank. Other genes $\left(1 \mathrm{~A}, 1 \mathrm{~B}^{\mathrm{Hel}}, 1 \mathrm{C}^{\mathrm{VPg}}\right.$, and $\left.1 \mathrm{D}^{\mathrm{Pro}}\right)$ were not considered in this study because of the present paucity of publically available sequence information. SatRNA nucleotide sequences were aligned with genomic RNA 5' UTRs with the Muscle algorithm (5) in Seaview (11), and these alignments were converted to FASTA files and uploaded to WebLogo3 to summarize sequence conservation (4) of the 5' UTR of satRNAs, genomic GFLV RNAs, genomic ArMV RNAs, and genomic GFLV, ArMV, and GDefV RNAs combined. Base compositions of nepovirus subgroup A genomic RNAs and satRNAs (excluding all gaps) were determined by Seaview and independence of base composition was tested with $\chi^{2}$ tests.

Phylogenetic trees were made and sequences statistically analyzed as previously described (1). Briefly, sequences were aligned using ClustalW (21) and manually curated in Seaview to maintain expected open reading frames (ORFs). The alignments were subjected to the 'find best nucleotide models' program option of MEGA5 for maximum likelihood trees, and phylogenetic trees were constructed using 5,000 bootstrap replications using the maximum likelihood method (39). Branches with less than $50 \%$ bootstrap support were collapsed. Genetic distance was calculated for each gene within and between virus species in MEGA5 using the maximum composite likelihood model. DnaSP (22) was used to conduct Tajima's neutrality test and to calculate Wright's $F_{\mathrm{ST}}$, Hudson's statistics $K_{\mathrm{ST}}{ }^{*}$ and $S_{\mathrm{nn}}$ for each gene within and between virus species. DataMonkey software (19) set to SLAC default parameters was used to discover all nonsynonymous (dN) and synonymous (dS) mutations and $\mathrm{dN} / \mathrm{dS}$ ratio from GFLV and ArMV alignments of each gene. Protein masses and isoelectric points were computed from the open reading frame of satRNAs using the Protean software in the Lasergene 9 genetic analysis package (DNASTAR, Madison, WI).

Transfer of GFLV isolates carrying satRNAs from grapevine tissue to $C$. quinoa. Frozen GFLV-infected grapevine leaves (R1 through R11) from Lodi, CA, were ground in inoculation

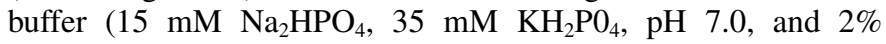
nicotine) and crude extract was pestle-inoculated onto four-leaved C. quinoa plants dusted with corundum. Forty-five to fifty-five C. quinoa were inoculated per GFLV isolate. Uninoculated apical leaves were tested for systemic infection by ELISA 20 days after inoculation. Plants were characterized for the presence of a satRNA by IC-RT-PCR as described above. The partial RNA2 of GFLV isolate R6 from grape and isolates R6-18 and R6-40 from 
C. quinoa was amplified by IC-RT-PCR and sequenced using primers G34 and G37, and G38 and G39. Each strain used in this study was passaged twice on $C$. quinoa before sequencing or use in experiments.

Effects of satRNAs on GFLV multiplication and symptoms in C. quinoa. GFLV strains F13 (42), FF (41), and R6-40 and R618 (this study) were used to test biological effects of the satRNA. Strain F13 contains a satRNA $(9,13,33)$ while strain FF does not (41). GFLV R6-18 and R6-40 were obtained from passaging infected grapevine tissue of isolate R6 from Lodi, CA to C. quinoa. Independent experiments were carried out to test the effect of satRNAs on either virus multiplication or symptom effects. Each experiment was repeated once. All plants were randomized on a greenhouse bench and the identities of each treatment concealed through the course of the experiment. C. quinoa were grown to the four leaf stage and mechanically inoculated with crude sap of infected $C$. quinoa leaves as described above. The greenhouse was maintained at $28^{\circ} \mathrm{C}$ with a $16-\mathrm{h}$ day length.

Groups of 20 (virus multiplication) or 10 (symptomatology) plants were inoculated with each isolate. For the virus multiplication experiment, five plants were sampled at 4, 7, 13, and 20 days postinoculation (dpi) and tested by DAS-ELISA. For the symptom experiment, symptoms were rated twice daily for 9 days followed by once daily for 14 days, from 4 to $26 \mathrm{dpi}$. Six symptom categories were noted including apical leaf curling (category 1 ), crumpling (category 2), vein clearing (category 3 ), expandedleaf flecking (category 4), yellowing/mottling (category 5), and lateral leaf vein banding (category 6). At $27 \mathrm{dpi}$, after conclusion of symptom analysis, each plant's above ground fresh weight and height were recorded, and above ground portions were dried in a cool greenhouse for 2 weeks at which time dry weight was recorded.

Statistical analyses of symptom, physiological, and virus titer experiments. Statistical tests were conducted in SAS (SAS Institute, Cary, NC) for the virus accumulation experiment and the experiment that tested the satRNA effect on symptoms and physiology. Each plant inoculated with a given virus strain was considered a replicate. Each data set was subjected to ANOVA followed by Tukey as a post-hoc test using the GLM procedure in SAS. For the symptom study, data for the six symptom categories was converted into a binary value (1 for presence of the symptom, and 0 for absence of the symptom), and the values were summed for each plant at each time point and this number was considered symptom severity. Area under the disease progress curve (AUDPC) was calculated for symptom severity over time (18). Virus strains were compared for AUDPC, plant height, plant fresh weight, and plant dry weight.

\section{RESULTS}

Grapevine leaf collection and GFLV satRNA detection and sequencing. Grapevine leaves were collected from 83 vines showing symptom characteristics of GFLV, e.g., foliar mosaic, chlorosis deformation, and shortened internodes, in three naturally infected vineyards in Lodi, CA in 2010 and 2012. The presence of GFLV was confirmed in symptomatic samples by DAS-ELISA and a satRNA was detected by IC-RT-PCR in GFLV-infected leaf samples from only one of the three vineyards surveyed. The satRNA was scattered throughout this vineyard but, among the 25 five-vine blocks where multiple GFLV-infected leaf samples were taken, nine blocks contained vines where all samples tested positive for the satRNA, seven contained only vines that tested negative for the satRNA, and nine contained vines that tested positive or negative for the satRNA. An unusual angular mosaic symptom was observed in one area of the vineyard containing satRNAs, although typical GFLV symptoms were observed throughout the vineyard, but presence of the satRNA did not correlate to this unusual GFLV symptom given its widespread distribution throughout the field (data not shown).

DNA amplicons of GFLV satRNA obtained by IC-RT-PCR from 11 leaf samples were gel extracted, cloned, and sequenced. SatRNA nucleotide sequences from Lodi, CA showed at least $94 \%$ identity with each other, but only up to $78 \%$ with the satRNA of GFLV-F13. Additionally, a GFLV isolate from the USDA-Plant Genetic Resource Unit (PGRU) in Geneva, NY, "PGRU accession 106", had a satRNA with 94 to $98 \%$ identity at the nucleotide level with satRNAs from Lodi, CA and $77.5 \%$ with the GFLV-F13 satRNA.

The full-length nucleotide sequence of the satRNA associated with GFLV isolates R6 and R2 from Lodi, CA was determined. They are each 1,140 nts long, compared with 1,114 nts of GFLVF13 and 1,092 to 1,139 nts of ArMV satRNAs. The GFLV satRNAs from Lodi, CA have a 24-nt 5' UTR, 78-nt 3' UTR, and a single ORF of 1,038 nts. The full-length sequences of the satRNAs associated with GFLV isolates R2 and R6 were deposited in GenBank as accessions KC162000 and KC161999, respectively.

Phylogenetic and sequence analysis. A maximum likelihood tree of GFLV and ArMV large satRNAs was constructed using TBRV large satRNAs $\mathrm{C}$ and $\mathrm{E}$ as outgroups (Fig. 1). The cladogram shows a clustering of the GFLV satRNAs from Lodi, CA (R2 and R6) with satRNAs of ArMV strains J86 and NW. A second clade has satRNAs of GFLV-F13, ArMV-Lilac, -P116, -P119, and -Hop (Fig. 1). SatRNAs associated with GFLV-R2 and -R6, and ArMV-J86 and -NW show less genetic distance overall or relative to each other than members of the other clade. Interestingly, the GFLV-F13 satRNA appears to share more recent ancestry with ArMV-Hop, -Lilac, -P116, and -P119 satRNAs than GFLV-R2 and -R6 satRNAs. Phylogenetic sequence relationships reflect overall sequence similarities and suggest that large satRNAs of subgroup A nepoviruses do not have a defined lineage based on identity of the helper virus.

In order to ascertain functional and evolutionary aspects of the GFLV satRNA, full-length nucleotide sequence alignments of GFLV and ArMV satRNAs and genes $1 \mathrm{E}^{\mathrm{Pol}}, 2 \mathrm{~A}^{\mathrm{HP}}, 2 \mathrm{~B}^{\mathrm{MP}}$, or $2 \mathrm{C}^{\mathrm{CP}}$ were compared. SatRNAs from either GFLV or ArMV show

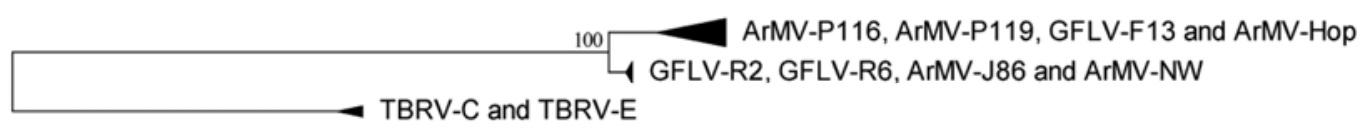

0.5

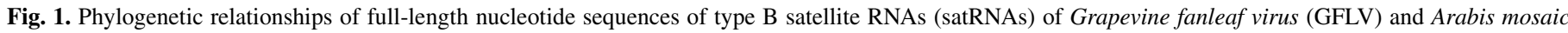

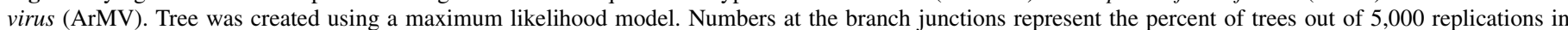

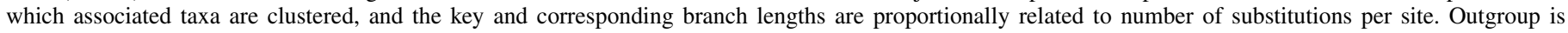

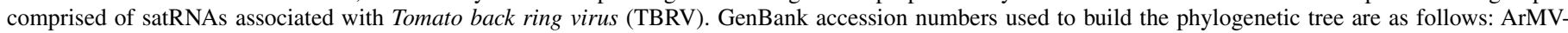

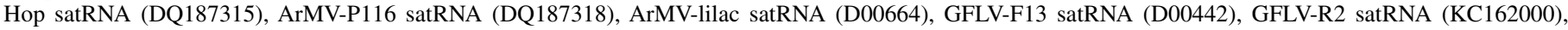

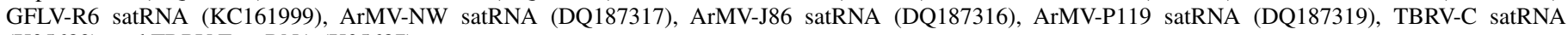
(X05689), and TBRV-E satRNA (X05687). 
greater intraspecific genetic distance than genes $1 \mathrm{E}^{\mathrm{Pol}}, 2 \mathrm{~A}^{\mathrm{HP}}$, $2 \mathrm{~B}^{\mathrm{MP}}$, or $2 \mathrm{C}^{\mathrm{CP}}$ (Table 1). SatRNAs show comparable interspecific distance with respect to genes $1 \mathrm{E}^{\mathrm{Pol}}, 2 \mathrm{~A}^{\mathrm{HP}}, 2 \mathrm{~B}^{\mathrm{MP}}$, but less than $2 \mathrm{C}^{\mathrm{CP}}$ which is sensu stricto, the gene that defines GFLV/ArMV speciation (6). By using Tajima's D as a measure of the frequency and distribution of sequence polymorphisms in a gene to infer its microevolutionary history, no significant nonrandom distribution of sequence polymorphisms was obtained for the satRNAs, indicating that the sequence composition cannot be attributed to population expansion or decline. Tajima's D was significantly large for gene $2 \mathrm{~B}^{\mathrm{MP}}$, which could suggest population expansion or balancing selection within ArMV and GFLV populations, although this result is likely attributable to the prevalence of interspecies GFLV/ArMV recombinants within this gene (38).

In order to better understand the interspecific diversity of satRNAs, sequence alignments were subjected to statistical tests for comparative relationships (Table 2). $K_{\mathrm{ST}}{ }^{*}$, which compares within-group diversity to total diversity (17), signaled genes $2 \mathrm{~B}^{\mathrm{MP}}$ and $2 \mathrm{C}^{\mathrm{CP}}$ possess significant species subdivision, whereas genes $1 \mathrm{E}^{\mathrm{Pol}}$ and $2 \mathrm{~A}^{\mathrm{HP}}$ and the satRNA do not. The nearest-neighbor sequence statistic $\left(S_{\mathrm{nn}}\right)(16)$ showed that while genes $1 \mathrm{E}^{\mathrm{Pol}}, 2 \mathrm{~A}^{\mathrm{HP}}$, $2 \mathrm{~B}^{\mathrm{MP}}$, and $2 \mathrm{C}^{\mathrm{CP}}$ are highly likely to have their most closely related sequences from the same virus species, the satRNAs nearest neighbors are less likely to be from the same virus species (Table 2 ). The satRNA $F_{\text {ST }}$ showed that only $4 \%$ of genetic variation is attributable to whether it belongs to GFLV or ArMV, whereas other genes showed 36 to $63 \%$ of their variation arising from their GFLV or ArMV identities. All together, three measures of intraversus interpopulation diversity showed the GFLV and ArMV satRNAs are not differentiated based on the identity of their helper virus, which suggests no species-specific identity for these satRNAs.

The ORFs of the GFLV satRNA from Lodi, CA correspond to a translation product of 346 amino acids, compared with 341 amino acids for GFLV-F13 satRNA (13), and 338 to 360 amino acids for ArMV satRNAs (43). The GFLV-R2 and -R6 satRNA-encoded proteins have a predicted molecular weight of $37 \mathrm{kDa}$ and an isoelectric point of 10.4, near the median of predictions for other GFLV and ArMV satRNA-encoded peptides. The ratio of nonsynonymous to synonymous nucleotide substitutions is higher for the satRNA-encoded proteins than for GFLV and ArMV proteins $1 \mathrm{E}^{\mathrm{Pol}}, 2 \mathrm{~A}^{\mathrm{HP}}, 2 \mathrm{~B}^{\mathrm{MP}}$, or $2 \mathrm{C}^{\mathrm{CP}}$ (Table 3 ), indicating the satRNA undergoes less negative selection than gene counterparts in its helper viruses. Twenty sites within the satRNA ORF were identified as having significant $(P<0.05)$ evidence for negative selection and none of the sites in the satRNA ORF showed significant $(P<0.05)$ evidence for positive selection. The fact that a large percentage of satRNA protein failed to be identified as under selection, relative to genes present on genomic RNAs, reflects the need for more satRNA sequences to gain a clearer picture of which sites are under negative or positive selection.

Relationships of $\mathbf{5}^{\prime}$-terminal nucleotide sequences of GFLV and ArMV satellite and genomic RNAs. Close inspection of the 5' UTR of the GFLV and ArMV satRNAs showed that they possess greater identity to their helper viruses' genomic RNAs than previously realized. While it has been previously noted that the first nucleotides of the GFLV-F13 satRNA were nearly identical to those of nepovirus genomic RNAs $(9,13)$, it is apparent that the first 148 to $155 \mathrm{nts}$ of the GFLV and ArMV satRNAs show significant identity to subgroup A nepovirus genomic RNAs' 5' UTRs (Fig. 2). The conservation is distributed across the first 137 to 191 nucleotides of GFLV, ArMV, or GDefV RNA1 or RNA2. The homologous area ends approximately at the uracil-rich tract in the genomic RNAs (nt 225 in Fig. 2). Much of the homologous sequence is contained within the ORF of the satRNAs (nts 15 to 25 and beyond) and shows six highly conserved stretches (motifs II-VII) of 8 to $15 \mathrm{nts}$ in addition to the first stretch of 12 conserved nucleotides (motif I) previously described $(9,13)$ (Fig. 2). The base compositions of the satRNAs and genomic RNAs are not significantly different before nt 226 in the alignment (Fig. 2) $(P=0.471)$ but are different after this nucleotide position $(P=$ $0.0014)$ according to $\chi^{2}$ analysis.

Passaging GFLV herbaceous hosts and curing the satRNA. Ten GFLV-infected grapevine leaf samples from Lodi, CA that contained a satRNA were used to inoculate $C$. quinoa. Infected $C$. quinoa isolates were produced from 6 of the 10 GFLV inocula, as shown by DAS-ELISA and characteristic vein clearing in apical leaves. Multiplex RT-PCR for GFLV RNA2 and the satRNA was performed using total RNA extracted from 10 infected $C$. quinoa. Inoculation with R6 resulted in two infected $C$. quinoa that differed in presence or absence of satRNA: R6-40 contained a satRNA while R6-18 did not. After two additional passages through $C$. quinoa, RT-PCR analysis confirmed presence or absence of the satRNA was maintained (Fig. 3). A partial GFLV RNA2 sequence was obtained for strains R6-18 and R6-40 in infected $C$. quinoa tissue and isolate R6 in grape tissue (GenBank accession number KC162001). The sequences, which include most of gene $2 \mathrm{~B}^{\mathrm{MP}}$, all of gene $2 \mathrm{C}^{\mathrm{CP}}$, and most of the $3^{\prime} \mathrm{UTR}$, are 95\% identical to GFLV isolate CACSB3 (GenBank accession number GU972578), which is from the same vineyard in Lodi, CA (41). The GFLV-R6-18 and GFLV-R6-40 RNA2 sequences were $99.6 \%$ identical at the nucleotide level, which showed that

TABLE 2. Genetic parameters of genes $1 \mathrm{E}^{\mathrm{Pol}}, 2 \mathrm{~A}^{\mathrm{HP}}, 2 \mathrm{~B}^{\mathrm{MP}}, 2 \mathrm{CP}$, and the satRNA within and between Grapevine fanleaf virus (GFLV) and Arabis mosaic virus (ArMV) isolates

\begin{tabular}{lcccc}
\hline & \multicolumn{4}{c}{$\begin{array}{c}\text { Comparative genetic parameters } \\
\text { between GFLV and ArMV populations }\end{array}$} \\
\cline { 2 - 5 } Gene & $K_{\text {st }}{ }^{*}$ & $P$ value & $S_{\text {nn }}$ & $F_{\text {st }}$ \\
\hline $1 E^{\mathrm{Pol}}$ & 0.05890 & $0.3821 \mathrm{~ns}$ & 1 & 0.53509 \\
$2 \mathrm{~A}^{\mathrm{HP}}$ & 0.06710 & $0.3950 \mathrm{~ns}$ & 0.98958 & 0.44919 \\
$2 \mathrm{~B}^{\mathrm{MP}}$ & 0.02868 & $0.0000^{* * *}$ & 0.98653 & 0.36131 \\
$2 \mathrm{C}^{\mathrm{CP}}$ & 0.02073 & $0.0271^{*}$ & 1 & 0.62785 \\
SatRNA & 0.00224 & $0.3423 \mathrm{~ns}$ & 0.77778 & 0.04177 \\
\hline
\end{tabular}

${ }^{a} K_{\mathrm{st}}{ }^{*}$ and associated $P$ value, $S_{\mathrm{nn}}$, and $F_{\mathrm{st}}$ statistics are shown for each gene between GFLV and ArMV isolate populations. Statistical significance is represented by one $(P<0.05)$ or three $(P<0.001)$ asterisks.

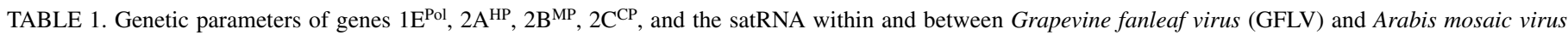
(ArMV) isolates

\begin{tabular}{|c|c|c|c|c|c|c|}
\hline \multirow[b]{3}{*}{ Gene } & \multicolumn{6}{|c|}{ Genetic distance and test of neutrality within and between groups ${ }^{\mathrm{a}}$} \\
\hline & \multicolumn{2}{|c|}{ GFLV } & \multicolumn{2}{|c|}{ ArMV } & \multicolumn{2}{|c|}{ ArMV vs. GFLV } \\
\hline & Genetic distance & Tajima's D & Genetic distance & Tajima's D & Genetic distance & Tajima's D \\
\hline $1 \mathrm{E}^{\mathrm{Pol}}$ & 0.043 & $-0.470 \mathrm{~ns}$ & 0.092 & $\mathrm{n} / \mathrm{a}(n=2)$ & 0.181 & $-0.914 \mathrm{~ns}$ \\
\hline $2 \mathrm{~A}^{\mathrm{HP}}$ & 0.056 & $-1.170 \mathrm{~ns}$ & 0.134 & $-1 \mathrm{~ns}$ & 0.203 & $-1.085 \mathrm{~ns}$ \\
\hline $2 \mathrm{~B}^{\mathrm{MP}}$ & 0.053 & $2.75^{*}$ & 0.107 & $2.36^{*}$ & 0.201 & $2.669 *$ \\
\hline $2 \mathrm{C}^{\mathrm{CP}}$ & 0.045 & $-0.741 \mathrm{~ns}$ & 0.099 & $-0.954 \mathrm{~ns}$ & 0.422 & $-1.0245 \mathrm{~ns}$ \\
\hline SatRNA & 0.167 & $\mathrm{n} / \mathrm{a}(n=3)$ & 0.256 & $-0.55 \mathrm{~ns}$ & 0.212 & $-0.57 \mathrm{~ns}$ \\
\hline
\end{tabular}

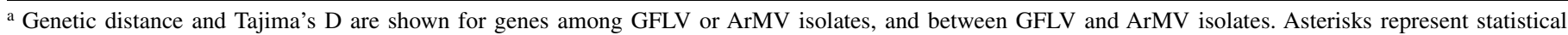
significance $(P<0.05)$ and "ns" represents lack of statistical significance according to DnaSP algorithm; n/a, not applicable because of small sample size $(n)$. 
the two strains established on $C$. quinoa were nearly identical except for the presence of absence of the satRNA, and these strains also showed similar identity to GFLV from the grapevine source from which they originated. Similarly, cDNAs of viral RNA progeny derived from F13 transcripts show 99.8\% nucleotide identity to the wild-type GFLV-F13. Thus, two sets of GFLV sister strains (R6-40 and R6-18, and F13 and FF) differing in presence or absence of satRNAs were created and these were used to characterize the biological effect of the satRNA.

Effect of the satRNA on GFLV accumulation and symptoms in C. quinoa. The GFLV-F13 and GFLV-R6 satRNAs were used to determine whether a satRNA has a measurable impact on its helper virus or on the systemic host $C$. quinoa. Viral protein accumulation was measured by DAS-ELISA at 4, 7, 13, and 20 dpi (Fig. 4). The effect of time point was significant $(F=$ 105.03, $P<0.0001)$, but effect of the virus strain was not $(F=$ $1.30, P=0.2825)$, nor was the interaction of virus strain and time point $(F=0.76, P=0.6559)$. GFLV capsid accumulation increased successively at 4,7 , and $13 \mathrm{dpi}$, and these differences were statistically significant $(P<0.05)$. Virus accumulation at 20 dpi was not significantly different from 13 dpi but was significantly different from all other time points. Presence or absence

TABLE 3. Genetic parameters of genes $1 \mathrm{E}^{\mathrm{Pol}}, 2 \mathrm{~A}^{\mathrm{HP}}, 2 \mathrm{~B}^{\mathrm{MP}}, 2 \mathrm{C}^{\mathrm{CP}}$, and the satRNA within and between Grapevine fanleaf virus (GFLV) and Arabis mosaic virus (ArMV) isolates

\begin{tabular}{|c|c|c|c|c|c|c|c|c|}
\hline \multirow[b]{2}{*}{ Gene } & \multicolumn{8}{|c|}{ Estimates of selection pressures ${ }^{\mathrm{a}}$} \\
\hline & $\log (\mathrm{L})$ & Mean & No. of positive & $\%$ Positive & No. of negative & $\%$ Negative & No. of neutral & $\%$ Neutral \\
\hline $1 \mathrm{E}^{\mathrm{Pol}}$ & -12776.9 & 0.134241 & 0 & 0 & 228 & 27.67 & 596 & 72.33 \\
\hline $2 \mathrm{~A}^{\mathrm{HP}}$ & -16684.9 & 0.291038 & 5 & 1.76 & 138 & 48.59 & 141 & 49.65 \\
\hline $2 \mathrm{C}^{\mathrm{CP}}$ & -39521.8 & 0.078302 & 2 & 0.396 & 437 & 86.53 & 66 & 13.07 \\
\hline P3 ${ }^{\text {satellite }}$ & -5227.99 & 0.519967 & 0 & 0 & 20 & 6.15 & 305 & 93.85 \\
\hline
\end{tabular}

a Normalized dN/dS ratios (mean) for each gene of GFLV and ArMV and log likelihood (L) scores for fitted models. Number and percentage of sites under positive, negative, and neutral selection $(P<0.05)$ are noted.
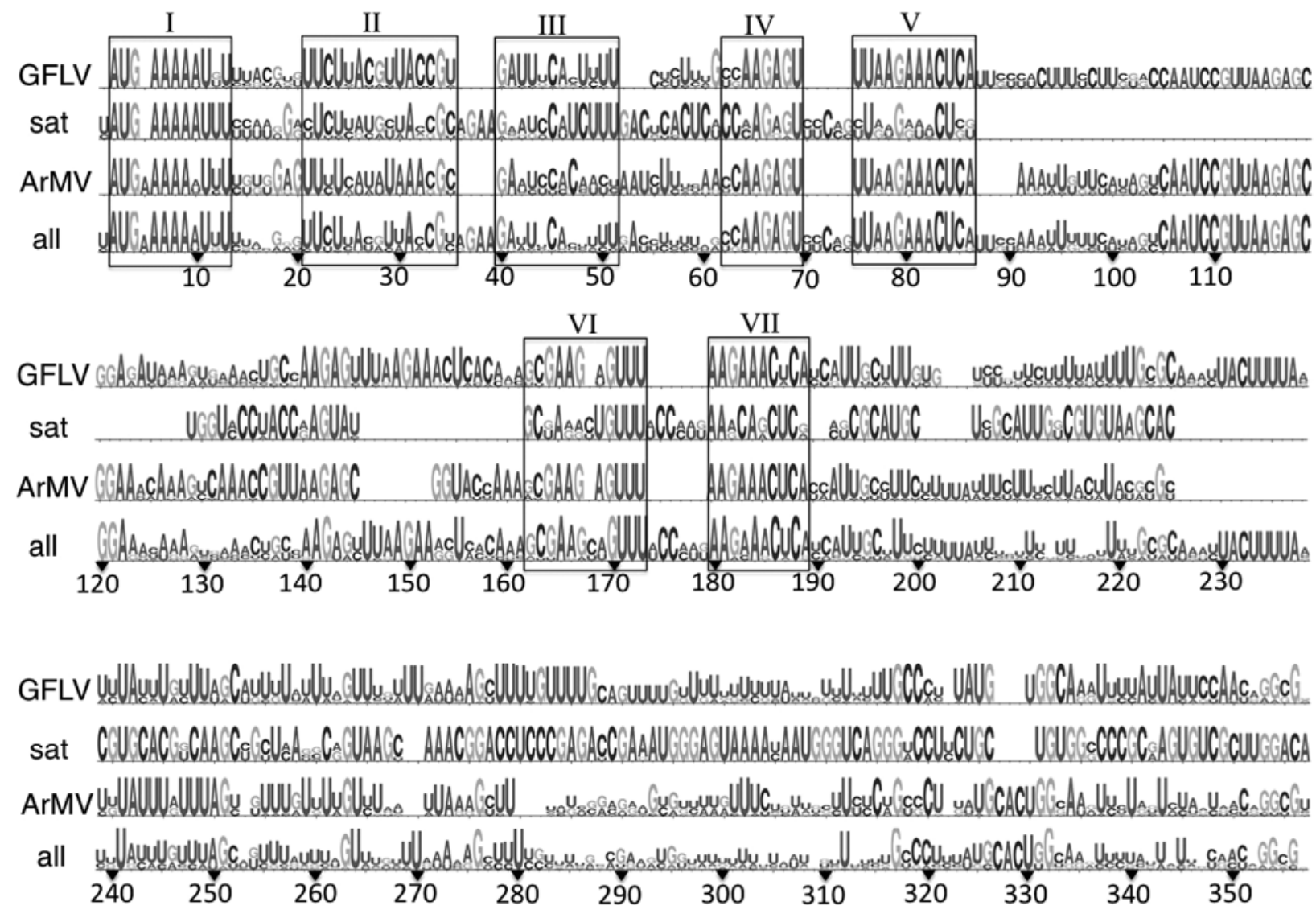

Fig. 2. Nucleotide sequence alignments at the $5^{\prime}$ region of nepovirus type B satellite RNAs (satRNAs) and subgroup A nepovirus genomic RNAs. WebLogo graphical display shows three blocks of sequence continuum, and boxed regions within blocks (I to VII) represent conserved nucleotide sequences among strains of Grapevine fanleaf virus (GFLV), subgroup A nepovirus large satRNAs, strains of Arabis mosaic virus (ArMV), and GFLV, satRNAs, ArMV, and Grapevine deformation virus (GDefV) (all). GenBank accession numbers used in the alignment are listed in the Figure 1 caption and as follows: ArMV-Lv RNA1 (EU617326), ArMV-ba RNA1I (GQ369526), ArMV-ba RNA1II (GQ369527), ArMV-ba RNA1III (GQ369528), ArMV-NW RNA1 (AY303786), GDefV RNA1 (NC_017939), GFLV-F13 RNA1 (D00915), GFLV-WAPN6132 RNA1 (GQ332373), GFLV-GFV1050-02 RNA1 (JX513889), GFLV-SAPSC3 RNA1 (JF968120), GFLV-WAPN173 RNA1 (GQ332372), ArMV-Lv RNA2 (EU617327), ArMV-ba RNA2IV (GQ369529), ArMV-ba RNA2V (GQ369530), ArMV-NW RNA2 (AY017339), ArMV-S RNA2U (X81814), ArMV-S RNA2L (X81815), ArMV-Ta RNA2 (EF426853), GDefV RNA2 (AY291208), GFLV-WAPN173 RNA2 (GQ332368), GFLV-NW RNA2 (AY027338), GFLV-SAPCS3 RNA2 (JF968121), GFLV-WAPN8133 RNA2 (GQ332369), GFLV-CACSB5 RNA2 (GU972580), GFLV-CACSC1 RNA2 (GU972581), GFLV-CAZINA4 RNA2 (GU972574), GFLV-F13 RNA2 (D00915), GFLV-GHu RNA2 (EF426852), GFLV-WACF2142 RNA2 (GQ332371), GFLV-WACH911 RNA2 (GQ332364), GFLV-WAPN57 RNA2 (GQ332367), GFLV-WAPN165 RNA2 (GQ332365), and GFLV-WAPN1492 RNA2 (GQ332370). 
of the satRNA did not significantly impact viral protein accumulation overall or at any time point. This indicates the GFLV satRNA did not discernably change the competitive host-virus interaction.

The four GFLV strains were inoculated to C. quinoa and symptoms were recorded at multiple time points after which plant physiological measurements were taken. Strains produced significant differences for each measurement including symptom severity over time (AUDPC) $(F=31.07, P<0.0001)$, height $(F=4.68$, $P=0.0073)$, above-ground fresh weight $(F=7.93, P=0.0003)$, and above-ground dry weight $(F=3.88, P=0.0168)$. The GFLV genomic RNAs had a striking impact on symptoms (Fig. 5), though not necessarily on whole-plant physiology (Fig. 6), and the satRNA had no striking impact on either. No significant differences in AUDPC were detected between GFLV strains FF and F13, or between GFLV strains R6-18 and R6-40, but each strain of the F13 background was significantly different from each

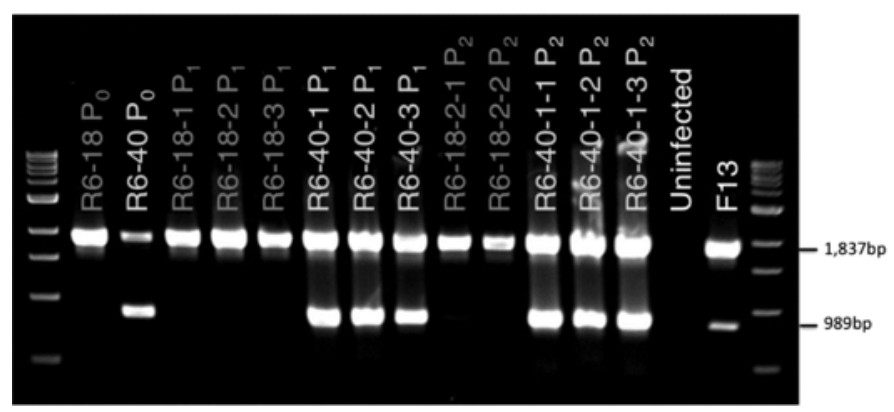

Fig. 3. Grapevine fanleaf virus (GFLV) strain R6 cured of its satellite RNA (satRNA). Duplex immunocapture-reverse transcription polymerase chain reaction for RNA2 and satRNA used total RNA extracted from Chenopodium quinoa subjected to three consecutive passages $\left(\mathrm{P}_{0}, \mathrm{P}_{1}\right.$, and $\left.\mathrm{P}_{2}\right) . \mathrm{P}_{0}$ denotes the initial passage from grapevine to $C$. quinoa plants 18 and 40 , and $\mathrm{P}_{1}$ and $\mathrm{P}_{2}$ denotes respective serial passages made from crude sap of initially infected plants. The RNA2 DNA amplicon is 1,837 bp and the satRNA amplicon is 989 bp. Total RNA from $C$. quinoa infected with GFLV-F13 was used as the positive control for RNA2 and satRNA, and total RNA from healthy $C$. quinoa constitutes the negative control. DNA fragments were resolved by electrophoresis on $1.5 \%$ agarose gels, and a 1-kb ladder (New England Biolabs) was used as a marker.

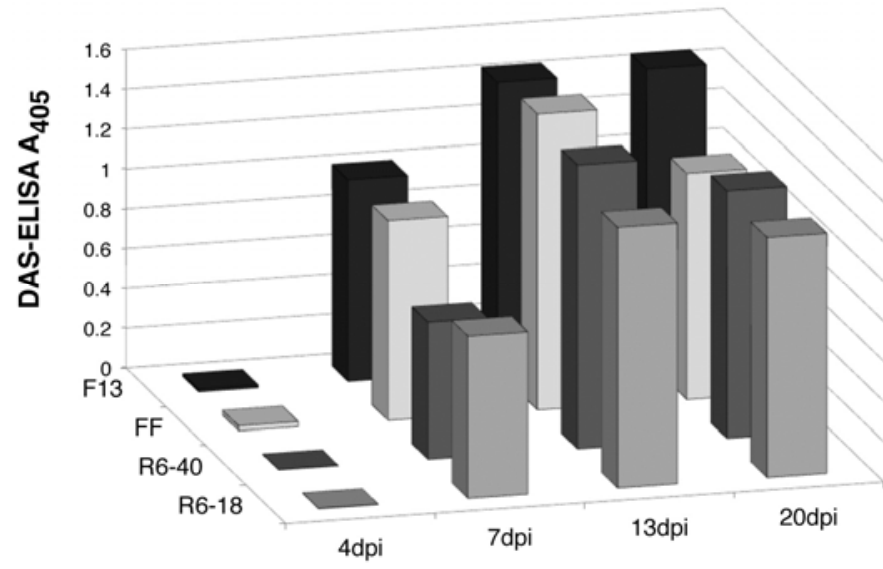

Fig. 4. Accumulation of Grapevine fanleaf virus (GFLV) capsid in mechanically inoculated Chenopodium quinoa. GFLV capsid was measured by double antibody sandwich-enzyme linked immunosorbent assay at an absorbance of $405 \mathrm{~nm}$, and means of five samples blanked are shown. GFLV strains F13 (black bars, with satRNA), FF (white bars, without satRNA), R6-40 (dark gray bars, with satRNA), and R6-18 (light gray bars, without satRNA) were measured at 4, 7, 13, and 20 days postinoculation (dpi). Significant differences for virus accumulation were detected between all time points except between 13 and $20 \mathrm{dpi}$. Differences in virus titer based on virus strain or virus strain by time point were not significant in analysis of variance tests. strain of the R6 background (Fig. 5). We detected significant differences between GFLV strains for three plant physiological parameters including height, dry weight and fresh weight. The only statistically significant physiological difference observed between sister strains was that fresh weight of FF was higher than that of F13, as well as R6-18 and R6-40 (Fig. 6). Except that FFinfected plants produced higher fresh weight than F13-infected plants, satRNAs did not have a significant measurable impact on C. quinoa symptoms or plant physiology.

\section{DISCUSSION}

GFLV satRNAs that are genetically similar were characterized by IC-RT-PCR and sequencing from vines in a naturally infected vineyard in Lodi, CA, as well as a vine in a USDA-PGRU grape germplasm collection in Geneva, NY. Based on the discovery of a very similar satRNA from disparate sources, records related to the USDA-PGRU accession were searched to attempt to trace the natural history of this satRNA. The GFLV- and satRNA-containing vine at USDA-PGRU (accession 106) is a selection of 'Grande Glabre', which was introduced to the United States from the Germplasm Repository in Bordeaux, France in 1962, kept at the USDA-Animal Plant Health Inspection Service Plant Pest Quarantine station in Beltsville, MD, for 5 years prior to introduction to the repository in Geneva, NY. Based on these records, PGRU accession 106 was likely infected at the time of introduction because GFLV and Xiphinema index, its ectoparasitic nematode vector (2), do not naturally occur in the eastern United States, including New York and Maryland, and pollen-transmission of GFLV is not known. The nucleotide sequence similarities suggest the satRNAs in Lodi, CA may share very recent ancestry with the satRNA that can be traced back to Bordeaux, France in the early 1960 s.

Field and laboratory observations support the hypothesis that the helper virus may easily dispense of its satRNA during plantto-plant transmission. The distribution of the satRNA among GFLV-infected vines in the naturally infected vineyard in Lodi, CA showed that the satRNA was absent from some GFLVinfected plants adjacent to others that contain the satRNA. Simi-

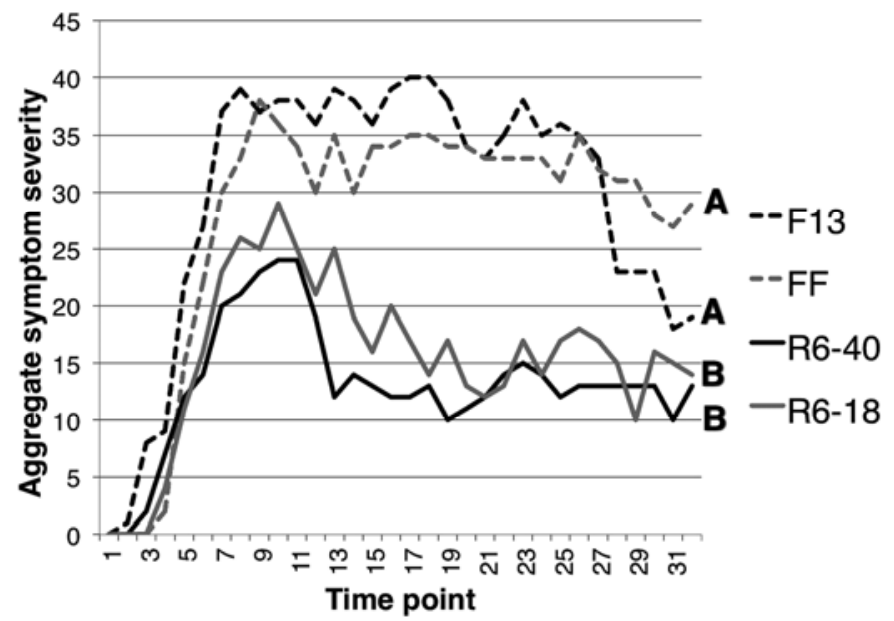

Fig. 5. Symptom severity over time of Grapevine fanleaf virus (GFLV) strains differing in genomic RNAs and satellite RNA (satRNA). Sum symptom severity on Chenopodium quinoa of GFLV strains F13 (with satRNA), FF (without satRNA), R6-40 (with satRNA), and R6-18 (without satRNA) ( $n=10)$ according to a six category rating scale, shown for 32 time points beginning at 4 days postinoculation. The first 18 time points represent twice daily ratings, and time points 19 through 34 represent once daily ratings. The height of the line graph represents the sum of all symptom categories among all ten replicates for each virus strain. Area under the disease progress curve corresponds to the total area under each line. The absence of significant difference according to Tukey $(P<0.05)$ is indicated with the same letters. 
larly, mechanical inoculation of crude sap from GFLV-infected grape leaves containing the satRNA resulted in a GFLV-infected plant that did not contain a satRNA (R6-18). This information suggests that plant-to-plant transmission in field and laboratory settings can result in loss of the satRNA. Conversely, detection of a satRNA in PGRU-106, which could only have been infected along with GFLV over 50 years prior, shows the satRNA can be stably maintained like its helper virus.

We did not detect an association between GFLV symptoms in a vineyard with presence of the satRNA. Saldarelli et al. (35) also reported no association between the satRNA and GFLV symptoms in diverse grapevine cultivars. Neither our study, nor Saldarelli et al. (35) evaluated the effect of the satRNA on symptoms and virus accumulation in a common grapevine cultivar. We tested for satRNA-induced symptom effects in a systemic host of GFLV, C. quinoa, but could not discern much impact of two diverse GFLV satRNAs on virus accumulation or symptoms. This was in contrast to a previous experiment showing the association

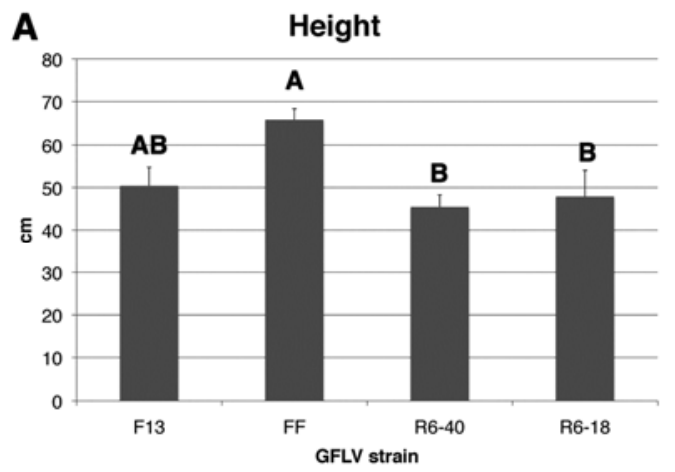

B Fresh weight

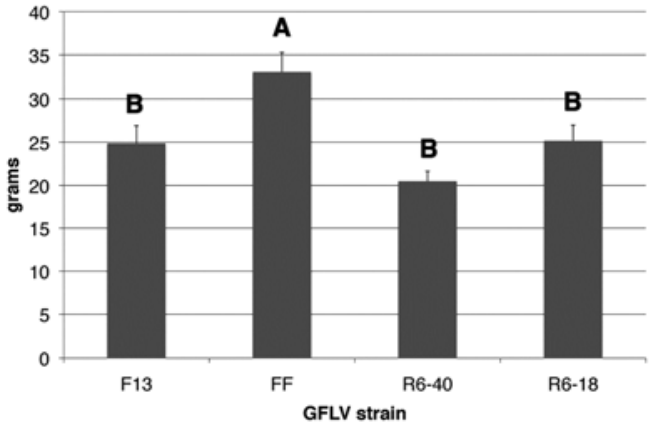

C Dry weight

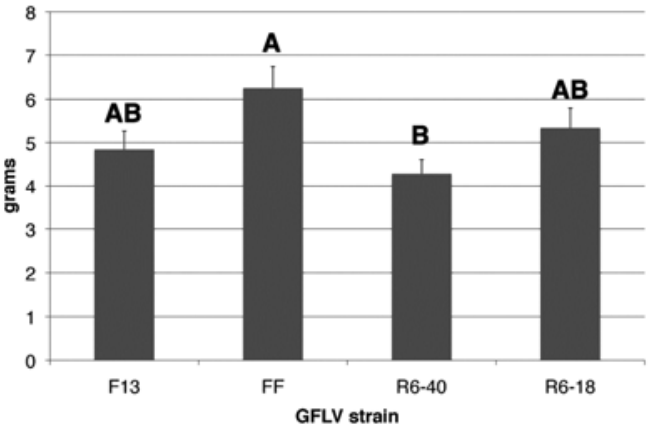

Fig. 6. Physiological parameters of Chenopodium quinoa infected with Grapevine fanleaf virus (GFLV) strains F13 (with satRNA), FF (without satRNA), R6-40 (with satRNA), and R6-18 (without satRNA) at 34 days postinoculation. Significant differences in A, height, $\mathbf{B}$, fresh weight, and $\mathbf{C}$, aboveground dry weight according to Tukey $(P<0.05)$ are represented with different letters within each graph. Columns represent the mean of 10 replicates and error bars represent standard error. of the ArMV-Lilac satRNA with an amelioration of symptoms in C. quinoa (25). Symptom measurements on C. quinoa showed greater differences between the two GFLV sister strains selected for this study than differences within GFLV sister strains differing in presence or absence of diverse satRNAs. Together, our data suggests the satRNA has a commensal relationship with GFLV and $C$. quinoa.

The origins of satRNAs are generally not known, but they may originate by chance recombination of viral and/or host nucleic acids (37). The type B satRNAs associated with subgroup A nepoviruses have substantial identity with genomic RNAs of the helper virus in their first 150 nucleotides. Based on this extended similarity, a recombination event likely occurred between the $5^{\prime}$ UTR of an ancestral subgroup A nepovirus RNA and another unidentified RNA with a putative break point at nucleotide 150 . Given that previous analyses of in vitro and in vivo translation products have demonstrated expression of the full-length satRNAencoded protein $(12,26,30)$, the recombination event appears to have given rise to satRNAs encoding a protein composed of an amino terminal region for which the corresponding nucleotide sequence is of a helper virus's 5' UTR. Because of an extended sequence relationship of this $5^{\prime}$ satRNA region specifically with ArMV, GFLV or GDefV, and because of the promiscuity of these satRNA associations, it appears satRNAs might have arisen in a common ancestor of these virus species and diversified in a nonhelper virus specific fashion. Currently, there is no nucleotide or amino acid sequence available in GenBank that shows continuous identity to the rest of the satRNA sequence beyond the first 150 nucleotides; therefore, the other parental RNA sequence remains unknown.

The evidence that satRNAs have diversified in a helper virus independent manner suggests certain conserved elements of the satRNA must be requisite for functionality in both GFLV and ArMV. Although the protein encoded by the satRNA is indispensable for satRNA replication by a GFLV or an ArMV helper $(13,26)$, the satRNA protein apparently undergoes less negative selection than other nepovirus genes. The conservation of nepovirus satRNAs and genomic RNAs $5^{\prime}$ ends well within the satRNA ORF suggests RNA replication determinants could be maintained within the satRNA $5^{\prime}$ UTR and $5^{\prime}$ end of its ORF. Like nepovirus satRNAs, the satellite RNA of Bamboo mosaic virus (satBaMV) possesses similarity to its helper virus in the $5^{\prime}$ untranslated region (23). This similarity is biologically significant since the BaMV RdRp specifically interacts with cis-acting elements in the satBaMV to initiate replication (15), and mutagenic analyses suggest this could also be true for nepovirus satRNAs $(13,14,24)$.

\section{ACKNOWLEDGMENTS}

Funding for J. Gottula came from a USDA National Institute of Food and Agriculture predoctoral fellowship and assistantships from the College of Agriculture and Life Sciences, Cornell University, through the New York State Agriculture Experiment Station. We thank P. MarsellaHerrick and M. Cheung for technical assistance and J. Oliver and E. Vigne for helpful discussions.

\section{LITERATURE CITED}

1. Alabi, O. J., Al Rwahnih, M., Karthikeyan, G., Poojari, S., Fuchs, M., Rowhani, A., and Naidu, R. A. 2011. Grapevine leafroll-associated virus 1 occurs as genetically diverse populations. Phytopathology 101:14461456.

2. Andret-Link, P., Laporte, C., Valat, L., Ritzenthaler, C., Demangeat, G., Vigne, E., Laval, V., Pfeiffer, P., Stussi-Garaud, C., and Fuchs, M. 2004. Grapevine fanleaf virus: Still a major threat to the grapevine industry. J. Plant Pathol. 86:183-195.

3. Collmer, C. W., and Howell, S. H. 1992. Role of satRNA in the expression of symptoms caused by plant viruses. Annu. Rev. Phytopathol. 30:419-442. 
4. Crooks, G. E., Hon, G., Chandonia, J. M., and Brenner, S. E. 2004. WebLogo: A sequence logo generator. Genome Res. 14:1188-1190.

5. Edgar, R. C. 2004. MUSCLE: Multiple sequence alignment with high accuracy and high throughput. Nucleic Acids Res. 32:1792-1797.

6. Elbeaino, T., Digiaro, M., Ghebremeskel, S., and Martelli, G. P. 2012. Grapevine deformation virus: Completion of the sequence and evidence on its origin from recombination events between Grapevine fanleaf virus and Arabis mosaic virus. Virus Res. 166:136-140.

7. Fritsch, C., and Mayo, M. 1993. Properties of the satRNA of nepoviruses. Biochimie 75:561-567.

8. Fuchs, M., Pinck, M., Etienne, L., Pinck, L., and Walter, B. 1991. Characterization and detection of Grapevine fanleaf virus by using cDNA probes. Phytopathology 81:559-565.

9. Fuchs, M., Pinck, M., Serghini, M. A., Ravelonandro, M., Walter, B., and Pinck, L. 1989. The nucleotide sequence of satRNA in Grapevine fanleaf virus, strain F13. J. Gen. Virol. 70:955-962.

10. Ghanem-Sabanadzovic, N. A., Sabanadzovic, S., Digiaro, M., and Martelli, G. P. 2005. Complete nucleotide sequence of the RNA-2 of Grapevine deformation and Grapevine Anatolian ringspot viruses. Virus Genes 3:335340.

11. Gouy, M., Guindon, S., and Gascuel, O. 2010. SeaView version 4: A multiplatform graphical user interface for sequence alignment and phylogenetic tree building. Mol. Biol. Evol. 27:221-224.

12. Hans, F., Fuchs, M., and Pinck, L. 1992. Replication of Grapevine fanleaf virus satRNA transcripts in Chenopodium quinoa protoplasts. J. Gen. Virol. 73:2517-2523.

13. Hans, F., Pinck, M., and Pinck, L. 1993. Location of the replication determinants of the satRNA associated with Grapevine fanleaf nepovirus (strain-F13). Biochimie 75:597-603.

14. Hemmer, O., Oncino, C., and Fritsch, C. 1993. Efficient replication of the in vitro transcripts from cloned cDNA of Tomato black ring virus satRNA requires the 48K satRNA-encoded protein. Virology 194:800-806.

15. Huang, Y. W., Hu, C. C., Lin, N. S., and Hsu, Y. H. 2010. Mimicry of molecular pretenders. RNA Biol. 7:1-10.

16. Hudson, R. R. 2000. A new statistic for detecting genetic differentiation. Genetics 155:2011-2014

17. Hudson, R. R., Boos, D. D., and Kaplan, N. L. 1992. A statistical test for detecting geographic subdivision. Mol. Biol. Evol. 9:138-151.

18. Jeger, M. J., and Viljanen-Rollinson, S. L. H. 2001. The use of the area under the disease-progress curve (AUDPC) to assess quantitative disease resistance in crop cultivars. Theor. Appl. Genet. 102:32-40.

19. Kosakovsky Pond, S. L., and Frost, S. D. W. 2005. Datamonkey: Rapid detection of selective pressure on individual sites of codon alignments. Bioinformatics 21:2531-2533.

20. Lamprecht, R. L., Maree, H., Stephan, D., Wetzel, T., and Burger, J. T. 2012. Molecular characterization of South African isolates of Grapevine fanleaf virus and an associated new satRNA. Pages 36-37 in: Proc. 17th Congress of the International Council for the Study of Virus and Viruslike Diseases of the Grapevine, Davis, CA.

21. Larkin, M. A., Blackshields, G., Brown, N. P., Chenna, R., McGettigan, P. A., McWilliam, H., Valentin, F., Wallace, I. M., Wilm, A., Lopez, R., Thompson, J. D., Gibson, T. J., and Higgins, D. G. 2007. Clustal W and Clustal X version 2.0. Bioinformatics 23:2947-2948.

22. Librado, P., and Rozas, J. 2009. DnaSP v5: A software for comprehensive analysis of DNA polymorphism data. Bioinformatics 25:1451-1452.

23. Lin, N. S., and Hsu, Y. H. 1994. A satRNA associated with Bamboo mosaic potexvirus. Virol. 202:707-714.

24. Liu, Y. L., Cooper, J. I., Coates, D., and Bauer, G. 1991. Biologically active transcripts of a large satRNA from Arabis mosaic nepovirus and the im- portance of 5' end sequences for its replication. J. Gen. Virol. 72:2867-2874.

25. Liu, Y. L., Cooper, J. I., Edwards, M. L., and Hellen, C. U. 1991. A satRNA of Arabis mosaic nepovirus and its pathological impact. Ann. Appl. Biol. 118:577-587.

26. Liu, Y. Y., and Cooper, J. I. 1993. The multiplication in plants of Arabis mosaic virus satRNA requires the encoded protein. J. Gen. Virol. 74:1471-1474

27. Liu, Y. Y., Hellen, C. U. T., Cooper, J. I., Bertioli, D. J., Coates, D., and Bauer, G. 1990. The nucleotide sequence of a satRNA associated with Arabis mosaic nepovirus. J. Gen. Virol. 71:1259-1263.

28. Martelli, G. P., and Boudon-Padieu, E. 2006. Directory of infectious diseases of grapevine and viruses and virus-like diseases of the grapevine. Option Méditerr. Série B. Studies Res. 55:35-50.

29. Mayo, M. A. 1991. Satellites. Classification and Nomenclature of Viruses. Fifth Report of the International Committee on Taxonomy of Viruses. R. I. B. Francki, C. M. Fauquet, D. L. Knudson, and F. Brown, eds. Arch. Virol. S2:400-402.

30. Moser, O., Fuchs, M., Pinck, L., and Stussi-Garaud, C. 1992. Immunodetection of Grapevine fanleaf virus satRNA-encoded protein in infected Chenopodium quinoa. J. Gen. Virol. 73:3033-3038.

31. Oliver, J. E., Vigne, E., and Fuchs, M. 2010. Genetic structure and molecular variability of Grapevine fanleaf virus populations. Virus Res. 152:30-40.

32. Oncino, C., Hemmer, O., and Fritsch, C. 1995. Specificity in the association of Tomato black ring virus satRNA with helper virus. Virology 213:87-96.

33. Pinck, L., Fuchs, M., Pinck, M., Ravelonandro, M., and Walter, B. 1988. A satRNA in Grapevine fanleaf virus strain F13. J. Gen. Virol. 69:233-239.

34. Roosinck, M. J., Sleat, D., and Palukaitis, P. 1992. Satellite RNAs of plant viruses: Structures and biological effects. Microbiol. Rev. 56:265-279.

35. Saldarelli, P., Minafra, A., and Walter, B. 1993. A survey of Grapevine fanleaf nepovirus isolates for the presence of satRNA. Vitis 32:99-102.

36. Sanfaçon, H., Wellink, J., Gall, O., Karasev, A., Vlugt, R., and Wetzel, T. 2009. Secoviridae: A proposed family of plant viruses within the order Picornavirales that combines the families Sequiviridae and Comoviridae, the unassigned genera Cheravirus and Sadwavirus, and the proposed genus Torradovirus. Arch. Virol. 154:899-907.

37. Simon, A., Roossinck, M., and Havelda, Z. 2004. Plant virus satellite and defective interfering RNAs: New paradigms for a new century. Annu. Rev. Phytopathol. 42:415-437.

38. Sokhandan-Bashir, N., and Melcher, U. 2012. Population genetic analysis of Grapevine fanleaf virus. Arch. Virol. 157:1919-1929.

39. Tamura, K., Peterson, D., Peterson, N., Stecher, G., Nei, M., and Kumar, S. 2011. MEGA5: Molecular evolutionary genetics analysis using maximum likelihood, evolutionary distance, and maximum parsimony methods. Mol. Biol. Evol. 28:2731-2739.

40. Vigne, E., Komar, V., and Fuchs, M. 2004. Field safety assessment of recombination in transgenic grapevines expressing the coat protein of Grapevine fanleaf virus. Transgenic Res. 13:165-179.

41. Viry, M., Serghini, M. A., Hans, F., Ritzenthaler, C., Pinck, M., and Pinck, L. 1993. Biologically active transcripts from cloned cDNA of genomic Grapevine fanleaf nepovirus RNAs. J. Gen. Virol. 74:169-174.

42. Vuittenez, A., Munck, I. C., and Kuszala, J. 1964. Souches de virus à haute aggressivité isolées de vignes atteintes de dégénérescence infectieuse. Etudes Virol. Appliquée 5:69-78.

43. Wetzel, T., Bassler, A., Amren, M. A. W., and Krczal, G. 2006. A RT/PCR-partial restriction enzymatic mapping (PREM) method for the molecular characterisation of the large satRNAs of Arabis mosaic virus isolates. J. Virol. Methods 132:97-103. 